\title{
Geometric and Meshing Properties of Conjugate Curves for Gear Transmission
}

\author{
Dong Liang, Bingkui Chen, Yane Gao, Shuai Peng, and Siling Qin \\ State Key Laboratory of Mechanical Transmission, Chongqing University, Chongqing 400044, China \\ Correspondence should be addressed to Bingkui Chen; bkchen@cqu.edu.cn
}

Received 23 June 2014; Revised 13 November 2014; Accepted 16 November 2014; Published 7 December 2014

Academic Editor: Evangelos J. Sapountzakis

Copyright (C) 2014 Dong Liang et al. This is an open access article distributed under the Creative Commons Attribution License, which permits unrestricted use, distribution, and reproduction in any medium, provided the original work is properly cited.

Conjugate curves have been put forward previously by authors for gear transmission. Compared with traditional conjugate surfaces, the conjugate curves have more flexibility and diversity in aspects of gear design and generation. To further extend its application in power transmission, the geometric and meshing properties of conjugate curves are discussed in this paper. Firstly, general principle descriptions of conjugate curves for arbitrary axial position are introduced. Secondly, geometric analysis of conjugate curves is carried out based on differential geometry including tangent and normal in arbitrary contact direction, characteristic point, and curvature relationships. Then, meshing properties of conjugate curves are further revealed. According to a given plane or spatial curve, the uniqueness of conjugated curve under different contact angle conditions is discussed. Meshing commonality of conjugate curves is also demonstrated in terms of a class of spiral curves contacting in the given direction for various gear axes. Finally, a conclusive summary of this study is given.

\section{Introduction}

The theory of plane or space curves and surfaces in the threedimensional Euclidean space forms the basis for development of differential geometry $[1,2]$. And its application in gear transmission, namely, the geometry theory of conjugate surfaces, has been widely applied to conventional gear drive [3-5]. Working performance of conjugate surfaces affects greatly the overall power and motion properties of gear drive. The mathematical principle, geometrical design, and characteristic analysis about conjugate surfaces were developed by many scholars.

Litvin et al. [6] proposed systematic methodology to study mathematical model of conjugate surfaces and analyzed the geometrical and meshing characteristics based on differential geometry. Chen [7] investigated surface geometry of spatial gear pairs and discussed general property from the practical point of view. Li [8] described spatial geometry modeling of conjugate surfaces. The specific application in engineering was also introduced. Di Puccio et al. [9] put forward a rather general formulation for generation and curvature analysis of conjugate surfaces via the alternative formulation of the theory of gearing. In [10], Wu and Luo studied a geometric theory of conjugate tooth surfaces and derived curvatures equations in terms of the limit functions of the first kind and considering the mating surfaces subjected to relative screw motion with constant translational and rotational velocities. Ito and Takahashi [11] analyzed curvatures in hypoid gears starting from a classical differential geometry point of view, but then introducing kinematic relationships. By employing the theory of screws, Dooner [12] provided the third law of gearing and formulated the limiting relationship between the radii of curvature of conjugate surfaces, which is valid only for the reference pitch surfaces. Duan et al. [13] presented the conjugate principle and basic characteristic of Bertrand conjugate surfaces. And Chen et al. [14] researched the geometric properties of moulding conjugate surfaces.

However, in some cases, the higher overload requirements are difficult to meet in existing conjugate surfaces. The convex-to-convex tooth profile is more common in contact pattern and it has low contact strength. In additional, there is larger sliding between general tooth surfaces which leads to the low transmission efficiency. Many studies have been carried out to develop various concepts, design, and analysis approaches toward these problems [15-21]. Generally speaking, the surface and curve are both common elements 
in nature. Compared with general surfaces, the contact between curves has more flexibility and diversity. The related investigations on conjugate curves have been carried out by the authors, and basic meshing principle and theoretical applications for gear transmission have been studied [22-25]. To further reveal general property of conjugate curves and extend the application in gear transmission, the geometric and meshing properties of conjugate curves are discussed in this paper.

The remainder of this paper is organized into four sections. In the following section, the principle descriptions of conjugate curves for arbitrary axial position are introduced. Based on differential geometry, geometric analysis of conjugate curves is carried out including tangent and normal in arbitrary contact direction, characteristic point, and curvature relationships in the next section. The subsequent section reveals meshing properties of conjugate curves: the uniqueness of conjugated curves and meshing commonality of conjugate curves. And a conclusive summary of this study is given in the last section.

\section{Principle Descriptions of Conjugate Curves for Arbitrary Axial Position}

Conjugate curves are described as two smooth curves that always keep continuous and tangent contact with each other in given contact direction under motion law. Particularly, the principle of conjugate curves was proposed only for parallelaxes gears in [22] and its procedure graph is displayed in Figure 1. However, for arbitrary axial position, the principle descriptions of conjugate curves are studied.

As shown in Figure 2, $S(O-x, y, z)$ and $S_{p}\left(O_{p}-x_{p}, y_{p}, z_{p}\right)$ are the fixed coordinate systems. Conjugate curves $\Gamma_{1}$ and $\Gamma_{2}$ are defined using coordinate systems $S_{1}\left(O_{1}-x_{1}, y_{1}, z_{1}\right)$ and $S_{2}\left(O_{2}-x_{2}, y_{2}, z_{2}\right)$ which are connected to pinion 1 and gear 2 , respectively. Point $P$ is the contact point.

The transformation matrix from coordinate systems $S_{1}$ to $S_{2}$ can be expressed as

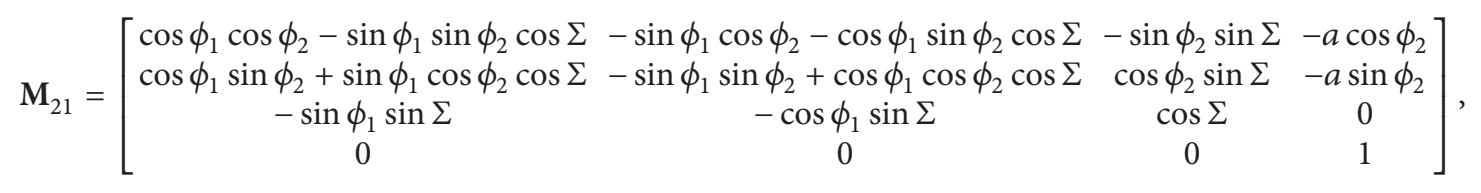

where $\phi_{1}$ and $\phi_{2}$ are the angular displacements of pinion 1 and gear 2 , respectively. $a$ is center distance and $\Sigma$ is angle between the axes of mating gears.

The parallel axis, intersecting axis, and crossed axis gear transmission can be realized separately by adjusting the center distance $a$ and angle $\Sigma$. Consider that the general curve $\Gamma_{1}$ is represented in parametric form as

$$
\Gamma_{1}: \mathbf{r}_{1}=x_{1}(\theta) \mathbf{i}_{1}+y_{1}(\theta) \mathbf{j}_{1}+z_{1}(\theta) \mathbf{k}_{1},
$$

where $\theta$ is curve parameter. $\mathbf{i}_{1}, \mathbf{j}_{1}$, and $\mathbf{k}_{1}$ are the unit vectors of coordinate system $S_{1}$.

The relative velocity of point $P^{(1)}$ of pinion 1 with respect to point $P^{(2)}$ of gear 2 is calculated as

$$
\begin{aligned}
\boldsymbol{v}_{1}^{(12)}= & -y_{1}\left(1+i_{21} \cos \Sigma\right)-z_{1} i_{21} \cos \phi_{1} \sin \Sigma \\
& \left.\quad-a i_{21} \sin \phi_{1} \cos \Sigma\right] \mathbf{i}_{1} \\
+ & {\left[x_{1}\left(1+i_{21} \cos \Sigma\right)+z_{1} i_{21} \sin \phi_{1} \sin \Sigma\right.} \\
& \left.\quad-a i_{21} \cos \phi_{1} \cos \Sigma\right] \mathbf{j}_{1} \\
+ & i_{21} \sin \Sigma\left(x_{1} \cos \phi_{1}-y_{1} \sin \phi_{1}-a\right) \mathbf{k}_{1},
\end{aligned}
$$

where $i_{21}$ is the transmission ratio and there exists $\phi_{2}=i_{21} \phi_{1}$.

Based on the curve trihedron established in [22], the normal vector to contact point in arbitrary direction of contact angle is expressed as

$$
\begin{aligned}
\mathbf{n}_{n}= & \left(t_{1} n_{\beta x_{1}}+t_{2} n_{\gamma x_{1}}\right) \mathbf{i}_{1}+\left(t_{1} n_{\beta y_{1}}+t_{2} n_{\gamma y_{1}}\right) \mathbf{j}_{1} \\
& +\left(t_{1} n_{\beta z_{1}}+t_{2} n_{\gamma z_{1}}\right) \mathbf{k}_{1},
\end{aligned}
$$

where $t_{1}$ and $t_{2}$ are the coefficients indicating the projection of contact angle $\alpha_{0}$ on the direction of principal normal and binormal vectors, respectively.

Moreover, meshing equation along given contact direction is derived as

$$
U \cos \phi_{1}-V \sin \phi_{1}=W
$$

where

$$
\begin{aligned}
& U=i_{21} \sin \Sigma\left(n_{n z 1} x_{1}-n_{n x 1} z_{1}\right)-i_{21} \cos \sum n_{n y 1} a \\
& V=-i_{21} \sin \Sigma\left(n_{n y 1} z_{1}-n_{n z 1} y_{1}\right)+i_{21} \cos \sum n_{n x 1} a \\
& W=\left(1+i_{21} \cos \Sigma\right)\left(n_{n x 1} y_{1}-n_{n y 1} x_{1}\right)+n_{n z 1} a i_{21} \sin \Sigma .
\end{aligned}
$$

Using transformation relation $\mathbf{r}_{2}=\mathbf{M}_{21} \mathbf{r}_{1}$ and meshing equation simultaneously, according to original curve $\Gamma_{1}$, the equation of conjugated curve $\Gamma_{2}$ is derived as (7). $\mathbf{r}_{2}$ is position vector of point $P$ in $S_{2}$. Considering transformation matrix from $S_{1}$ to $S$, the equation of line of action can also be obtained.

The comparison analysis of general principles of conjugate surfaces and conjugate curves is introduced. As the existing theoretical basis of gear geometry, the conjugate surfaces are widely used in the design and generation of tooth surfaces of gears. Usually, as shown in Figure 3, conjugated surface 2 can be obtained based on original surface 1 and the given motion law. Then the mating tooth surfaces of gear are generated [3].

However, the conjugate curves are described as two smooth curves that always keep continuous and tangent 


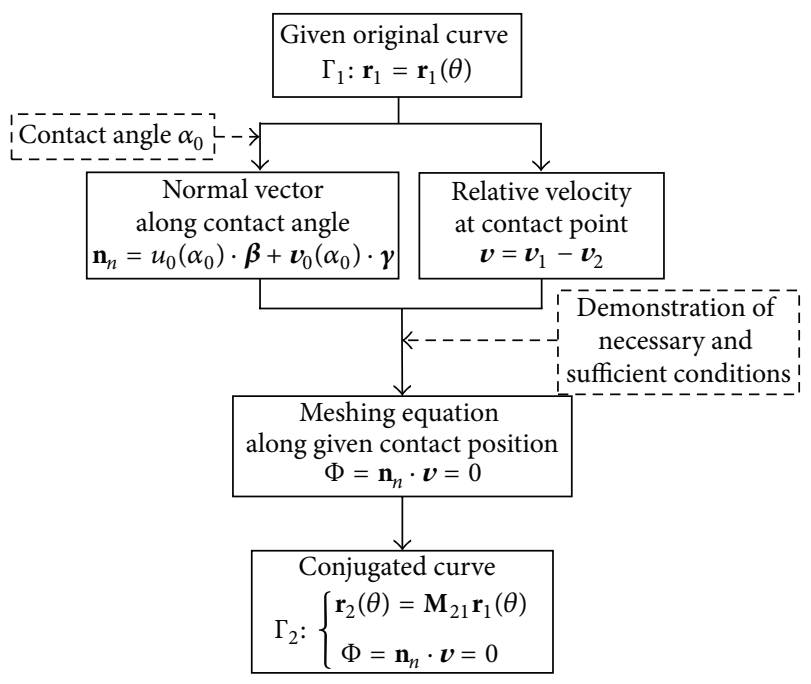

FIGURE 1: Principle procedure graph of conjugate curves.

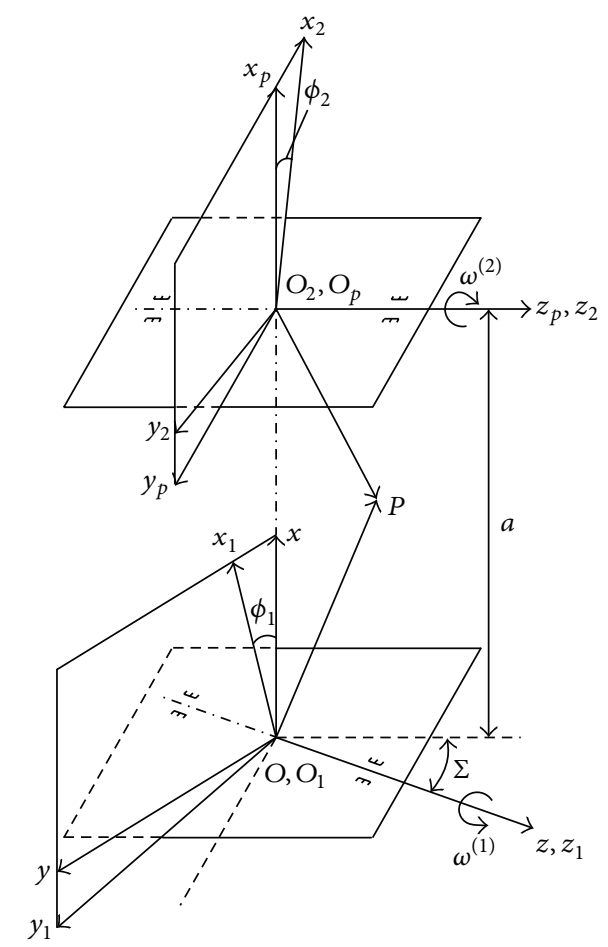

FIGURE 2: Coordinate systems for arbitrary axial position.

contact with each other in the given contact direction under motion law. Conjugated curve 2 can be derived according to original curve 1, designated contact direction, and given motion law. Then, the tubular meshing tooth surfaces inheriting meshing characteristics of the conjugate curves are generated based on equidistant-enveloping method [22, 23]. The generated tooth profiles specially can be diversity if choosing the different equidistant orientation and position. The theory of conjugate curves is simply represented in

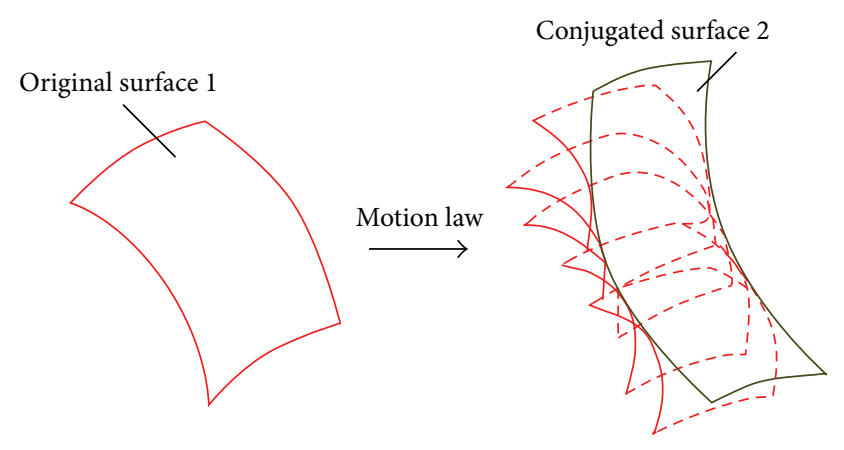

Figure 3: Theory of conjugate surfaces.

Figure 4 and three different contact models of tooth profiles are also displayed in Figure 5:

$$
\begin{aligned}
& x_{2}= x_{1}\left[\cos \phi_{1} \cos \left(i_{21} \phi_{1}\right)-\sin \phi_{1} \sin \left(i_{21} \phi_{1}\right) \cos \Sigma\right] \\
&+y_{1}\left[-\sin \phi_{1} \cos \left(i_{21} \phi_{1}\right)-\cos \phi_{1} \sin \left(i_{21} \phi_{1}\right) \cos \Sigma\right] \\
&-z_{1} \sin \left(i_{21} \phi_{1}\right) \sin \Sigma-a \cos \left(i_{21} \phi_{1}\right), \\
& y_{2}= x_{1}\left[\cos \phi_{1} \sin \left(i_{21} \phi_{1}\right)+\sin \phi_{1} \cos \left(i_{21} \phi_{1}\right) \cos \Sigma\right] \\
&+y_{1}\left[-\sin \phi_{1} \sin \left(i_{21} \phi_{1}\right)+\cos \phi_{1} \cos \left(i_{21} \phi_{1}\right) \cos \Sigma\right] \\
&+z_{1} \cos \left(i_{21} \phi_{1}\right) \sin \Sigma-a \sin \left(i_{21} \phi_{1}\right), \\
& z_{2}=-x_{1} \sin \phi_{1} \sin \Sigma-y_{1} \cos \phi_{1} \sin \Sigma+z_{1} \cos \Sigma, \\
& U \cos \phi_{1}-V \sin \phi_{1}=W .
\end{aligned}
$$

According to the aforementioned descriptions, the following analysis conclusions can be got as follows:

(1) The contact element is surface for the theory of conjugate surfaces, while curve is the contact element for the theory of conjugate curves.

(2) The tooth surfaces of conjugate-surface gear are generated based on the surface. But the tooth surfaces of conjugate-curve gear are developed by means of the spatial curve. The relation between the curve and surface is revealed.

(3) Conjugated surface 2 is unique for original surface 1 and the meshing pair of conjugate surfaces is also unique. However, conjugated curve 2 is unique in the arbitrary designated contact direction for original curve 1 and the mating conjugate curves are diversity if choosing the various contact direction.

(4) The carrier and form of curves are varied compared to that of surface. The ideal conjugate curves can be obtained according to the selection of original curve and determination of contact direction. The different meshing tooth surfaces containing the characteristics of conjugate curves can be generated. 


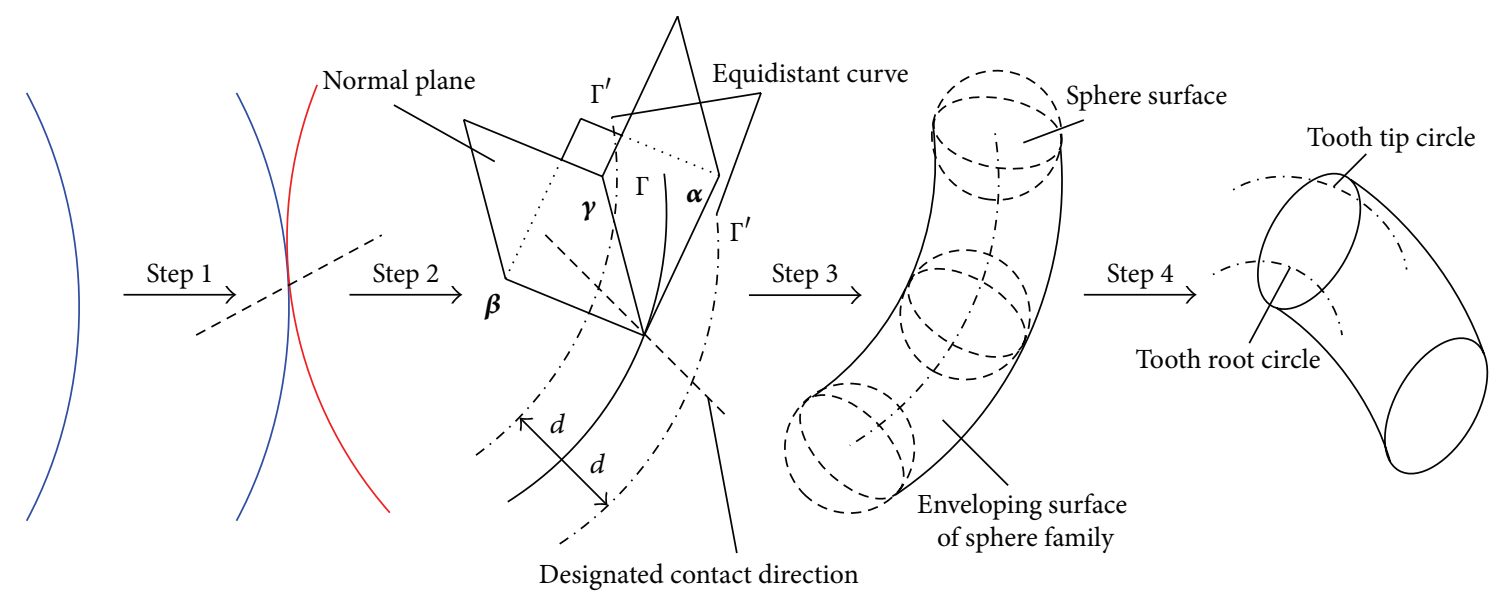

FIGURE 4: Theory of conjugate curves.

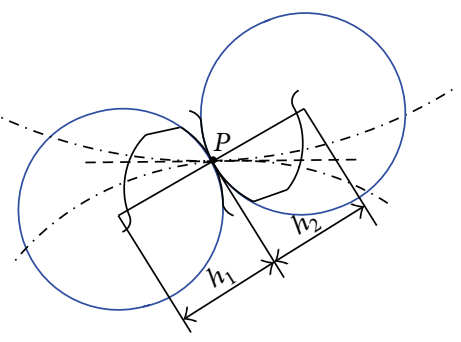

(a)

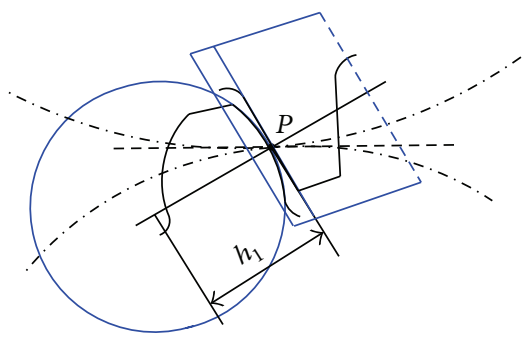

(b)

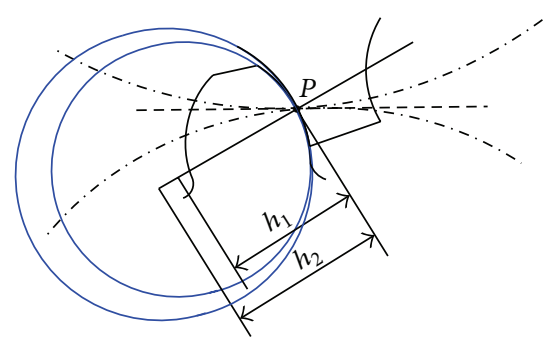

(c)

FIgURE 5: Three different contact models of gear: (a) convex-convex contact; (b) convex-plane contact; (c) convex-concave contact.

\section{Geometric Characteristic Analysis of Conjugate Curves}

3.1. Tangent and Normal to Conjugate Curves in Arbitrary Contact Direction. The concept of a tangent to the conjugate curves at contact point is similar to that of general curve based on the so-called limiting positions of rays [3]. Consider a set of rays that are drawn through a curve point $M$ and its neighboring points $M_{i}(i=1,2, \ldots, n)$. As points $M_{i}$ approach point $M$, all rays come to some limit position. In the case shown in Figure 6, there are two limiting rays with coinciding lines of action. These two rays form the tangent to the curve at point $M$ and it is identified as a regular point of the curve. A tangent $\mathbf{T}$ exists only at a regular point of a curve. A curve point where the tangent $\mathbf{T}$ does not exist or is equal to zero is identified as a singular point.

Then tangent $\mathbf{T}$ is determined and it has

$$
\mathbf{T}: \mathbf{r}_{\theta}=x^{\prime}(\theta) \mathbf{i}_{1}+y^{\prime}(\theta) \mathbf{j}_{1}+z^{\prime}(\theta) \mathbf{k}_{1}
$$

For conjugate curves $\Gamma_{1}$ and $\Gamma_{2}$, the tangents $\mathbf{T}_{1}$ and $\mathbf{T}_{2}$ are expressed by $\mathbf{r}_{1 \theta}, \mathbf{r}_{2 \theta}$, respectively.

The normal $\mathbf{n}_{n}$ to conjugate curves at contact point in arbitrary direction of contact angle is perpendicular to the tangent to the mating curves. There is an infinite number

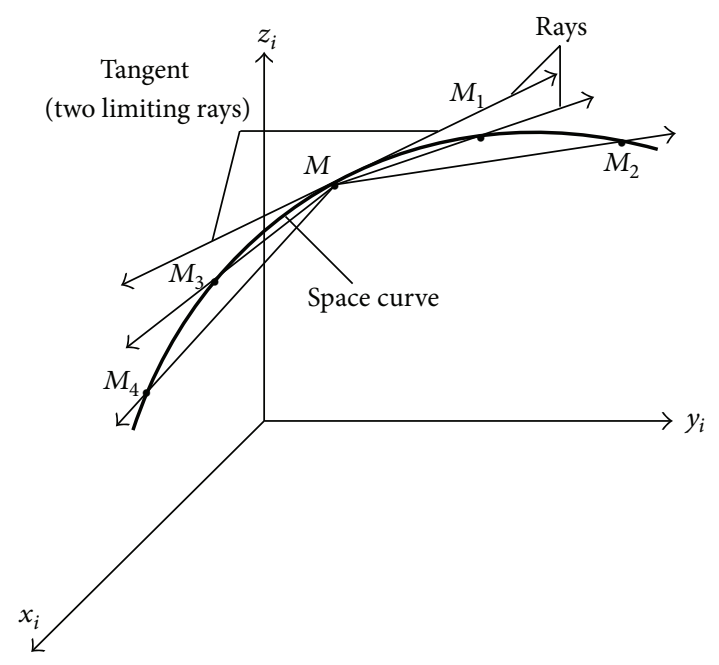

FIGURE 6: Illustration of rays and tangent.

of normals $\mathbf{n}$ belonging to normal plane at contact point $M$. For instance, vector $\mathbf{n}_{n}$ is one of the set of curve normals in Figure 7. Three orthogonal vectors can be determined: the tangent vector $\boldsymbol{\alpha}$, principal normal $\boldsymbol{\beta}$, and binormal $\boldsymbol{\gamma}$. 


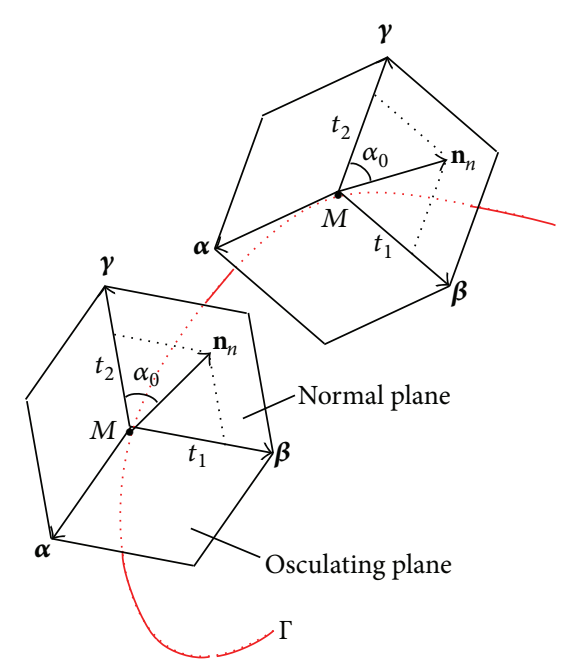

FIGURE 7: Normal to conjugate curves in arbitrary contact direction.

The principal normal vector $\boldsymbol{\beta}$ and binormal $\boldsymbol{\gamma}$ can be calculated, respectively, as

$$
\begin{aligned}
\boldsymbol{\beta}= & \frac{x_{\theta \theta}\left(y_{\theta}^{2}+z_{\theta}^{2}\right)-x_{\theta}\left(y_{\theta} y_{\theta \theta}+z_{\theta} z_{\theta \theta}\right)}{\left(x_{\theta}^{2}+y_{\theta}^{2}+z_{\theta}^{2}\right)^{2}} \mathbf{i}_{1} \\
& +\frac{y_{\theta \theta}\left(x_{\theta}^{2}+z_{\theta}^{2}\right)-y_{\theta}\left(x_{\theta} x_{\theta \theta}+z_{\theta} z_{\theta \theta}\right)}{\left(x_{\theta}^{2}+y_{\theta}^{2}+z_{\theta}^{2}\right)^{2}} \mathbf{j}_{1} \\
& +\frac{z_{\theta \theta}\left(x_{\theta}^{2}+y_{\theta}^{2}\right)-z_{\theta}\left(x_{\theta} x_{\theta \theta}+y_{\theta} y_{\theta \theta}\right)}{\left(x_{\theta}^{2}+y_{\theta}^{2}+z_{\theta}^{2}\right)^{2}} \mathbf{k}_{1}, \\
\gamma= & \frac{y_{\theta} z_{\theta \theta}-z_{\theta} y_{\theta \theta}}{\left(x_{\theta}^{2}+y_{\theta}^{2}+z_{\theta}^{2}\right)^{3 / 2}} \mathbf{i}_{1}-\frac{x_{\theta} z_{\theta \theta}-z_{\theta} x_{\theta \theta}}{\left(x_{\theta}^{2}+y_{\theta}^{2}+z_{\theta}^{2}\right)^{3 / 2}} \mathbf{j}_{1} \\
& +\frac{x_{\theta} y_{\theta \theta}-y_{\theta} x_{\theta \theta}}{\left(x_{\theta}^{2}+y_{\theta}^{2}+z_{\theta}^{2}\right)^{3 / 2}} \mathbf{k}_{1} .
\end{aligned}
$$

The normal vector to contact point in arbitrary direction of contact angle can be represented as a linear combination of principal normal and binormal vectors, and it has $\mathbf{n}_{n}=t_{1} \boldsymbol{\beta}+$ $t_{2} \gamma$. Here, contact angle $\alpha_{0}$ is defined as the angle between arbitrary normal vector $\mathbf{n}_{n}$ and binormal vector $\boldsymbol{\gamma}$.

3.2. Characteristic Point. Given the curve $\Gamma_{1}$, adding a motion parameter $\lambda$, the family of curves $\left\{\Gamma_{i}\right\}$ is obtained [2]. The enveloping curve $\Gamma_{2}$ can be generated as depicted in Figure 8 if satisfying the following conditions: (1) curve $\Gamma_{1}$ is smooth and regular curve. (2) At every instant $t, \Gamma_{1}$ and $\Gamma_{2}$ touch each other in contact point $M$. (3) Each point of curve $\Gamma_{2}$ is also a contact point at a unique instant $t$. Obviously, it is the set of different contact points. (4) The relation between curves $\Gamma_{1}$ and $\Gamma_{2}$ can be rendered quite symmetrical, and each may be said to be conjugated for the other if their domains are suitably restricted.

According to (2), family of curves $\left\{\Gamma_{i}\right\}$ can be expressed as

$$
\left\{\Gamma_{i}\right\}: \mathbf{r}_{1}=\mathbf{r}_{1}(\theta, \lambda) .
$$

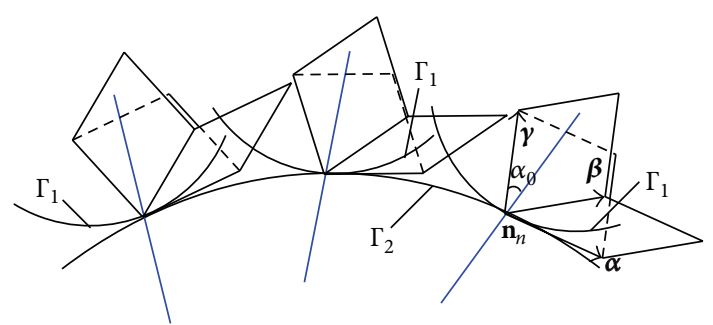

FIGURE 8: Envelope of a family of curves in given contact direction.

And if the enveloping curve exists, it has

$$
\overline{\mathbf{r}_{1}}=\overline{\mathbf{r}_{1}}(\theta(\lambda), \lambda)=\overline{\mathbf{r}_{1}}(\lambda) .
$$

For arbitrary curve in family of curves $\left\{\Gamma_{i}\right\}$, its tangent vector is written as

$$
\mathbf{r}_{1 \theta}=\frac{\partial \mathbf{r}_{1}}{\partial \theta}
$$

The calculation for enveloping curve is

$$
\overline{\mathbf{r}_{1}}=\frac{\partial \mathbf{r}_{1}}{\partial \theta} \frac{d \theta}{d \lambda}+\frac{\partial \mathbf{r}_{1}}{\partial \lambda}=\mathbf{r}_{1 \theta} \frac{d \theta}{d \lambda}+\mathbf{r}_{1 \lambda}
$$

There is common tangent between both curves at contact point so that it has $\mathbf{r}_{1 \theta} \| \overline{\mathbf{r}_{1}}$. Then

$$
\mathbf{r}_{1 \theta} \times \overline{\mathbf{r}_{1}}=0
$$

Substituting (12) and (13) into (14) yields the result as

$$
\mathbf{r}_{1 \theta} \times \overline{\mathbf{r}_{1}}=\mathbf{r}_{1 \theta} \times\left(\frac{\partial \mathbf{r}_{1}}{\partial \theta} \frac{d \theta}{d \lambda}+\frac{\partial \mathbf{r}_{1}}{\partial \lambda}\right)=\mathbf{r}_{1 \theta} \times \mathbf{r}_{1 \lambda}=0 ;
$$

that is,

$$
\mathbf{r}_{1 \theta} \times \mathbf{r}_{1 \lambda}=\left|\begin{array}{ccc}
\mathbf{i}_{1} & \mathbf{j}_{1} & \mathbf{k}_{1} \\
\frac{\partial x_{1}}{\partial \theta} & \frac{\partial y_{1}}{\partial \theta} & \frac{\partial z_{1}}{\partial \theta} \\
\frac{\partial x_{1}}{\partial \lambda} & \frac{\partial y_{1}}{\partial \lambda} & \frac{\partial z_{1}}{\partial \lambda}
\end{array}\right|=0
$$

So the mathematical formula of enveloping curve of family of curves $\left\{\Gamma_{i}\right\}$ is derived as

$$
\begin{gathered}
\mathbf{r}_{1}=\mathbf{r}_{1}(\theta, \lambda), \\
\mathbf{r}_{1 \theta} \times \mathbf{r}_{1 \lambda}=\Phi(\theta, \lambda)=0 .
\end{gathered}
$$

Compared with the solution of conjugated curve in Section 2, (17) has the same meaning with (7). Usually, each family of surfaces and its enveloping surface are not generally tangent at one point, but along a curve which is called the characteristic line on the surface. Actually it is also the contact curve for both surfaces proposed above. The enveloping surface could be used as the set of family of single parameter characteristic lines [3]. Similarly, each family of curves and its enveloping curve are tangent with point contact and the set 
of contact points which are called characteristic points finally forms the target curve.

According to aforementioned discussion, we can get such following conditions.

(1) The necessary and sufficient conditions for contact between arbitrary curve of family of curves $\left\{\Gamma_{i}\right\}$ and its enveloping curve are

$$
\Phi(\theta, \lambda)=\mathbf{r}_{1 \theta} \times \mathbf{r}_{1 \lambda}=0
$$

and the generation formula of enveloping curve is

$$
\begin{gathered}
\mathbf{r}_{1}=\mathbf{r}_{1}(\theta, \lambda), \\
\mathbf{r}_{1 \theta} \times \mathbf{r}_{1 \lambda}=\Phi(\theta, \lambda)=0 .
\end{gathered}
$$

(2) The characteristic point which is also the conjugate contact point with generated enveloping curve exists on each curve of family of curves $\left\{\Gamma_{i}\right\}$. It has

$$
\begin{gathered}
\mathbf{r}_{1}=\mathbf{r}_{1}\left(\theta, \lambda_{0}\right), \\
\mathbf{r}_{1 \theta} \times \mathbf{r}_{1 \lambda_{0}}=\Phi\left(\theta, \lambda_{0}\right)=0,
\end{gathered}
$$

where $\lambda_{0}$ is the constant.

3.3. Curvature Relationships. It is well known that the curvature is of great importance indicator for evaluating geometric characteristic of curve, and it reflects the bending degree at one point of curve. As the parameter of arc length, $s$ is introduced to the derivation of curve curvature. As shown in Figure 9, $M_{0}$ is arbitrary given point of curve $\Gamma$ and $M_{1}$ is its neighboring curve point. The correspondence parameters of arc length are $s_{0}$ and $s_{0}+\Delta s$, respectively. $\Delta \varphi$ is angle formed by the tangents taken at the given and neighboring curve points. $\Delta s$ is arc length between the neighboring points. Then the change rate of tangents when point $M_{0}$ turns the arc length $\Delta s$ can be described as the curvature of curve $\Gamma$ at this point.

The curvature $\kappa_{0}$ of a spatial curve is determined as

$$
\kappa_{0}=\lim _{\Delta s \rightarrow 0}\left|\frac{\Delta \varphi}{\Delta s}\right|,
$$

where the subscript " 0 " in $\kappa_{0}$ indicates that the curvature is considered for a small piece of the curve located in the osculating plane. Based on differential geometry, the curvature $\kappa_{0}$ can be calculated with formula $\kappa_{0}=\left|\mathbf{r}_{s} \times \mathbf{r}_{s s}\right|$.

To derive equations for determination of the curve curvature $\kappa_{0}$ and curve torsion $\tau$, the parameter $s$ of arc length and curve parameter $\theta$ are considered to be related by function $s(\theta)$. Based on (2), the curve to be discussed can be represented as $\mathbf{r}_{1}(s(\theta))$. Differentiation of this vector function yields

$$
\mathbf{r}_{1 \theta}=\mathbf{r}_{s} \frac{d s}{d \theta}
$$

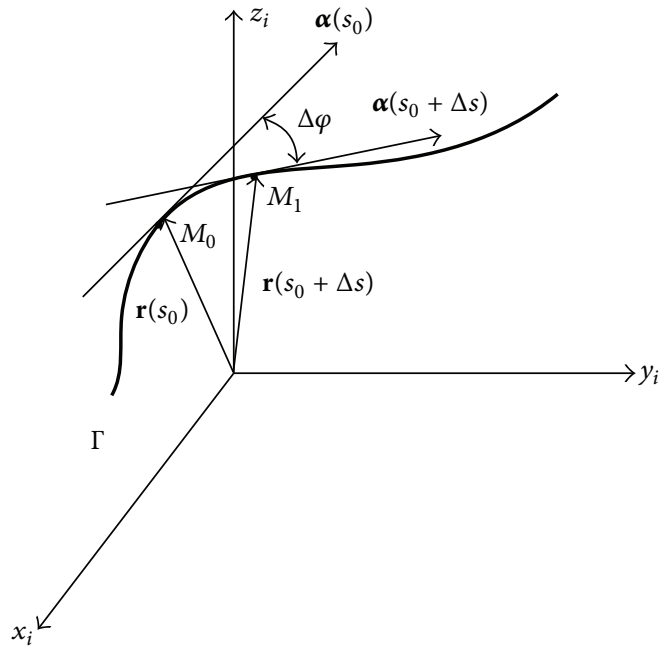

FIGURE 9: Curve curvature analysis.

Here $d s / d \theta=\left|\mathbf{r}_{1 \theta}\right|$ because $\left|\mathbf{r}_{s}\right|=1$. Moreover, it has

$$
\begin{aligned}
& \mathbf{r}_{1 \theta \theta}=\mathbf{r}_{s s}\left(\frac{d s}{d \theta}\right)^{2}+\mathbf{r}_{s}\left(\frac{d^{2} s}{d \theta^{2}}\right), \\
& \mathbf{r}_{1 \theta \theta \theta}=\mathbf{r}_{s s s}\left(\frac{d s}{d \theta}\right)^{3}+3 \mathbf{r}_{s s}\left(\frac{d s}{d \theta}\right)\left(\frac{d^{2} s}{d \theta^{2}}\right)+\mathbf{r}_{s}\left(\frac{d^{3} s}{d \theta^{3}}\right) .
\end{aligned}
$$

Substituting (22) and (23) into the expression of curvature $\kappa_{0}$, we obtain the simplified result

$$
\begin{gathered}
\mathbf{r}_{s} \times \mathbf{r}_{s s}=\frac{\mathbf{r}_{1 \theta} \times \mathbf{r}_{1 \theta \theta}}{(d s / d \theta)^{3}}=\frac{\mathbf{r}_{1 \theta} \times \mathbf{r}_{1 \theta \theta}}{\left|\mathbf{r}_{1 \theta}\right|^{3}}, \\
\kappa_{0}=\frac{\left|\mathbf{r}_{1 \theta} \times \mathbf{r}_{1 \theta \theta}\right|}{\left|\mathbf{r}_{1 \theta}\right|^{3}} \\
=\left[\left(x_{1 \theta} y_{1 \theta \theta}-x_{1 \theta \theta} y_{1 \theta}\right)^{2}+\left(x_{1 \theta} z_{1 \theta \theta}-x_{1 \theta \theta} z_{1 \theta}\right)^{2}\right. \\
\left.+\left(y_{1 \theta} z_{1 \theta \theta}-y_{1 \theta \theta} z_{1 \theta}\right)^{2}\right]^{1 / 2} \\
\times\left(\left(x_{1 \theta}^{2}+y_{1 \theta}^{2}+z_{1 \theta}^{2}\right)^{3 / 2}\right)^{-1} .
\end{gathered}
$$

For the analysis of torsion of curve, the formula $\tau=$ $\left(\mathbf{r}_{s}, \mathbf{r}_{s s}, \mathbf{r}_{s s s}\right) / \kappa_{0}^{2}$ yields

$$
\tau=\frac{\left(\mathbf{r}_{1 \theta} \times \mathbf{r}_{1 \theta \theta}\right) \cdot \mathbf{r}_{1 \theta \theta \theta}}{\left(\mathbf{r}_{1 \theta} \times \mathbf{r}_{1 \theta \theta}\right)^{2}} .
$$

\section{Meshing Characteristics of Conjugate Curves}

4.1. Uniqueness of Conjugated Curve. The conjugated curve is generated in terms of the meshing equation and transformation matrixes among different coordinate systems, according to a given original curve. The normal vector $\mathbf{n}_{n}$ to conjugate curves at contact point in arbitrary direction of contact angle 


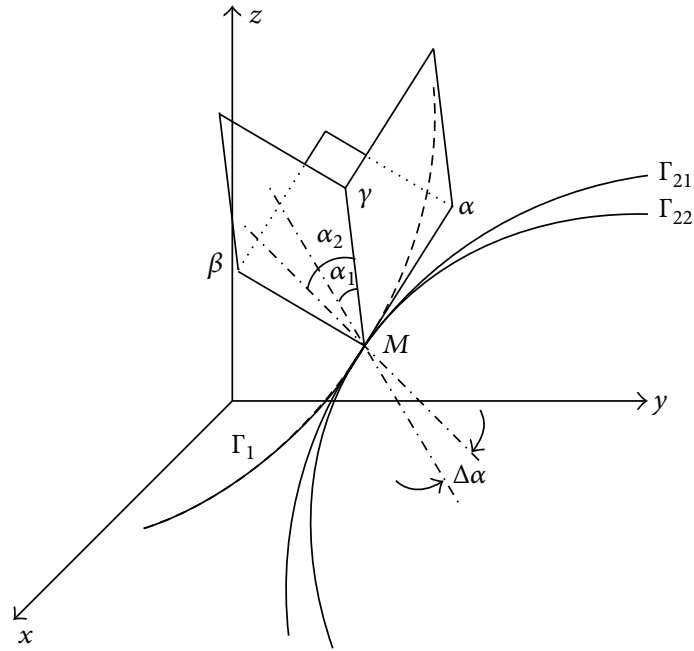

FIGURE 10: Two conjugated curves for different contact angles $\alpha_{1}, \alpha_{2}$.

can be determined in the previous study. Particularly, from the expression of normal vector $\mathbf{n}_{n}$, we can identify that coefficients $t_{1}$ and $t_{2}$ indicate the projection of contact angle $\alpha_{0}$ on the direction of principal normal and binormal vectors, respectively.

Given the different coefficients $t_{1}$ and $t_{2}$, if the original curve is determined, the corresponding conjugated curve can be represented in various results. Then the further study on the relationship between obtained conjugated curves is carried out. Given the contact angles $\alpha_{1}, \alpha_{2}$ (where $\alpha_{2}=\alpha_{1}+$ $\Delta \alpha$ ) in Figure 10, respectively, based on principle descriptions of conjugate curves in Section 2, the conjugated curves $\Gamma_{2 i}$ $(i=1,2)$ which are separately related to given conditions are expressed as

$$
\begin{aligned}
x_{2}^{(i)}= & x_{1}\left[\cos \phi_{1}^{(i)} \cos \left(i_{21} \phi_{1}^{(i)}\right)-\sin \phi_{1}^{(i)} \sin \left(i_{21} \phi_{1}^{(i)}\right) \cos \Sigma\right] \\
& +y_{1}\left[-\sin \phi_{1}^{(i)} \cos \left(i_{21} \phi_{1}^{(i)}\right)\right. \\
& \left.-\cos \phi_{1}^{(i)} \sin \left(i_{21} \phi_{1}^{(i)}\right) \cos \Sigma\right] \\
& -z_{1} \sin \left(i_{21} \phi_{1}^{(i)}\right) \sin \Sigma-a \cos \left(i_{21} \phi_{1}^{(i)}\right) \\
y_{2}^{(i)}= & x_{1}\left[\cos \phi_{1}^{(i)} \sin \left(i_{21} \phi_{1}^{(i)}\right)+\sin \phi_{1}^{(i)} \cos \left(i_{21} \phi_{1}^{(i)}\right) \cos \Sigma\right] \\
& +y_{1}\left[-\sin \phi_{1}^{(i)} \sin \left(i_{21} \phi_{1}^{(i)}\right)\right. \\
& \left.+\cos \phi_{1}^{(i)} \cos \left(i_{21} \phi_{1}^{(i)}\right) \cos \Sigma\right] \\
& +z_{1} \cos \left(i_{21} \phi_{1}^{(i)}\right) \sin \Sigma-a \sin \left(i_{21} \phi_{1}^{(i)}\right) \\
z_{2}^{(i)}= & -x_{1} \sin \phi_{1}^{(i)} \sin \Sigma-y_{1} \cos \phi_{1}^{(i)} \sin \Sigma+z_{1} \cos \Sigma, \\
\phi_{1}^{(i)}= & \arcsin \frac{W^{(i)}}{\sqrt{U^{(i)}}+V^{(i)}{ }^{2}}-\arctan \frac{V^{(i)}}{U^{(i)}}
\end{aligned}
$$

Considering that conjugated curve $\Gamma_{21}$ rotates about central contact point $M$ with angle $\Delta \alpha$, it has this expression after the coordinate transformation as

$$
\begin{aligned}
& x_{2}^{(1) \prime}=x_{2}^{(1)} \cos \Delta \alpha+y_{2}^{(1)} \sin \Delta \alpha, \\
& y_{2}^{(1) \prime}=-x_{2}^{(1)} \sin \Delta \alpha+y_{2}^{(1)} \cos \Delta \alpha, \\
& z_{2}^{(1) \prime}=z_{2}^{(1)} .
\end{aligned}
$$

Substituting (26) into (27), the derived result is written as

$$
\begin{aligned}
x_{2}^{(1) \prime}= & \left(x_{1} A_{1}+y_{1} B_{1}-z_{1} C_{1}\right) \cos \Delta \alpha \\
& +\left(x_{1} A_{2}+y_{1} B_{2}+z_{1} C_{2}\right) \sin \Delta \alpha \\
& -a \cos \left(\Delta \alpha-i_{21} \phi_{1}^{(1)}\right) \\
y_{2}^{(1) \prime}= & \left(-x_{1} A_{1}-y_{1} B_{1}+z_{1} C_{1}\right) \sin \Delta \alpha \\
& -\left(x_{1} A_{2}-y_{1} B_{2}-z_{1} C_{2}\right) \cos \Delta \alpha \\
& -a \sin \left(\Delta \alpha-i_{21} \phi_{1}^{(1)}\right), \\
z_{2}^{(1) \prime} & =-x_{1} \sin \phi_{1}^{(1)} \sin \Sigma-y_{1} \cos \phi_{1}^{(1)} \sin \Sigma+z_{1} \cos \Sigma,
\end{aligned}
$$

where

$$
\begin{aligned}
& A_{1}=\cos \phi_{1}^{(1)} \cos \left(i_{21} \phi_{1}^{(1)}\right)-\sin \phi_{1}^{(1)} \sin \left(i_{21} \phi_{1}^{(1)}\right) \cos \Sigma ; \\
& A_{2}=\cos \phi_{1}^{(1)} \sin \left(i_{21} \phi_{1}^{(1)}\right)+\sin \phi_{1}^{(1)} \cos \left(i_{21} \phi_{1}^{(1)}\right) \cos \Sigma ; \\
& B_{1}=-\sin \phi_{1}^{(1)} \cos \left(i_{21} \phi_{1}^{(1)}\right)-\cos \phi_{1}^{(1)} \sin \left(i_{21} \phi_{1}^{(1)}\right) \cos \Sigma ; \\
& B_{2}=-\sin \phi_{1}^{(1)} \sin \left(i_{21} \phi_{1}^{(1)}\right)+\cos \phi_{1}^{(1)} \cos \left(i_{21} \phi_{1}^{(1)}\right) \cos \Sigma ; \\
& C_{1}=\sin \left(i_{21} \phi_{1}^{(1)}\right) \sin \Sigma ; \\
& C_{2}=\cos \left(i_{21} \phi_{1}^{(1)}\right) \sin \Sigma .
\end{aligned}
$$

A comparison about the expressions of $\Gamma_{22}$ and $\Gamma_{21}^{\prime}$ between (26) and (28) is worked. It can be concluded from the simplified results that the two conjugated curves $\Gamma_{21}$ and $\Gamma_{22}$ are the same and unique curves, while the difference is that $\Gamma_{22}$ is the rotational curve of $\Gamma_{21}$ about contact point $M$ under given angle $\Delta \alpha$. For verifying above conclusion, the mathematical example for parallel axis gearing based on cylindrical spiral curve is introduced and its parametric form is represented as

$$
\mathbf{r}_{1}=R \cos \theta \mathbf{i}_{1}+R \sin \theta \mathbf{j}_{1}+p \theta \mathbf{k}_{1},
$$

where $R$ is the radius of pitch circle, $\theta$ is spiral curve parameter, and $p$ is helix parameter. Through the aforementioned principle of conjugate curves, the equation of conjugated curve is derived as

$$
\begin{aligned}
& x_{2}=(R-a) \cos \left(i_{21} \theta\right), \\
& y_{2}=-(R-a) \sin \left(i_{21} \theta\right), \\
& z_{2}=p \theta .
\end{aligned}
$$




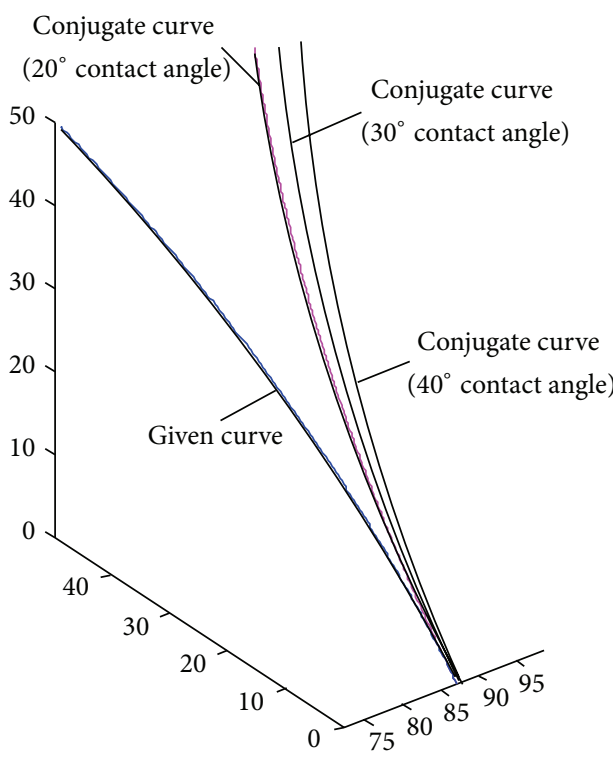

(a)

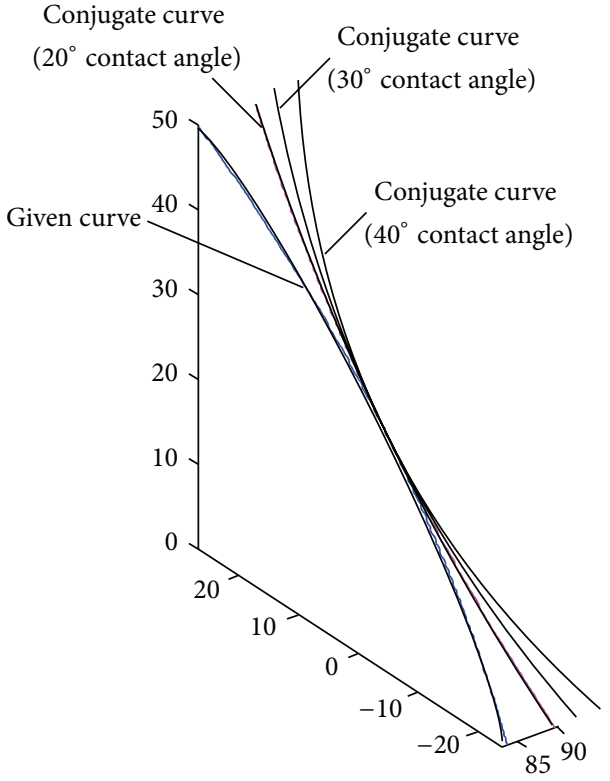

(b)

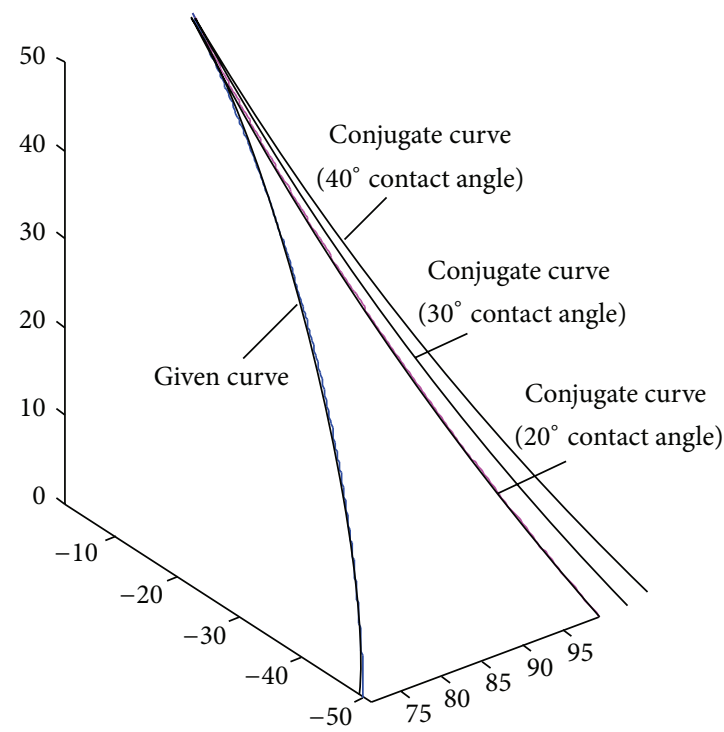

(c)

FIGURE 11: Uniqueness of conjugated curve at any contact position: (a) beginning position; (b) middle position; (c) end position.

A simplified meshing model of conjugate curves along various contact angles for gear drive is developed by means of Matlab software, as displayed in Figure 11, according to the designated parameters shown in Table 1.

From Figure 11, the generated conjugated curves are different if the selected contact angles are various. When the gear pair rotates with fixed angular velocity, the movement of conjugate curves with 20 degrees rotation, 30 degrees rotation, and 40 degrees rotation can be described, respectively. The engagement point is still fixed on the common point during the whole meshing. Throughout this process, contact point changes gradually in the axial direction, and the line of action is always a straight line which is parallel with gear axes. The characteristics of conjugate curves provide more flexibility for the design of gear transmission. We can choose the optimized design result which is suitable for practical application in terms of appropriate parameters.

4.2. Meshing Commonality of Conjugate Curves. To analyze intrinsic nature of conjugate curves, a class of spiral curves contacting in the given direction for various gear axes is discussed.

4.2.1. Gear Pair with Parallel Axis. Assuming that parameters $a \neq 0$ and $\Sigma=0$, the gear pair with parallel axis can be obtained, as displayed in Figure 12. A calculation procedure 


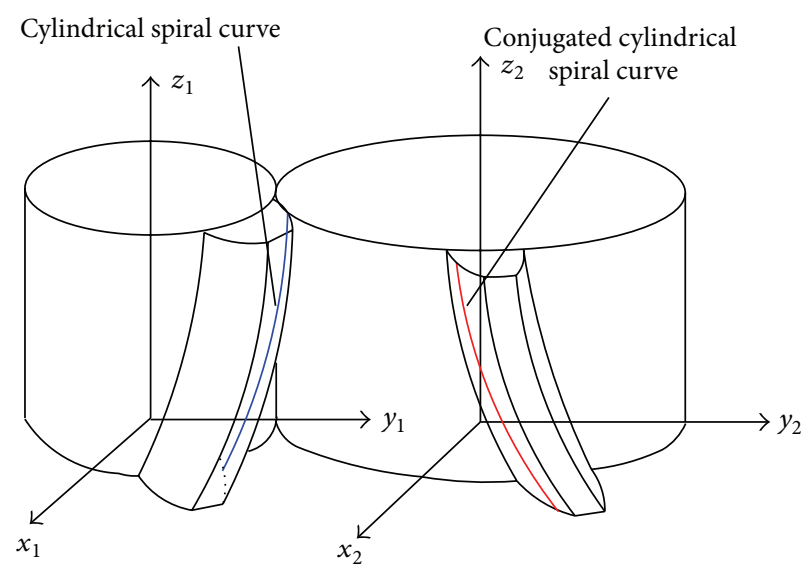

(a)

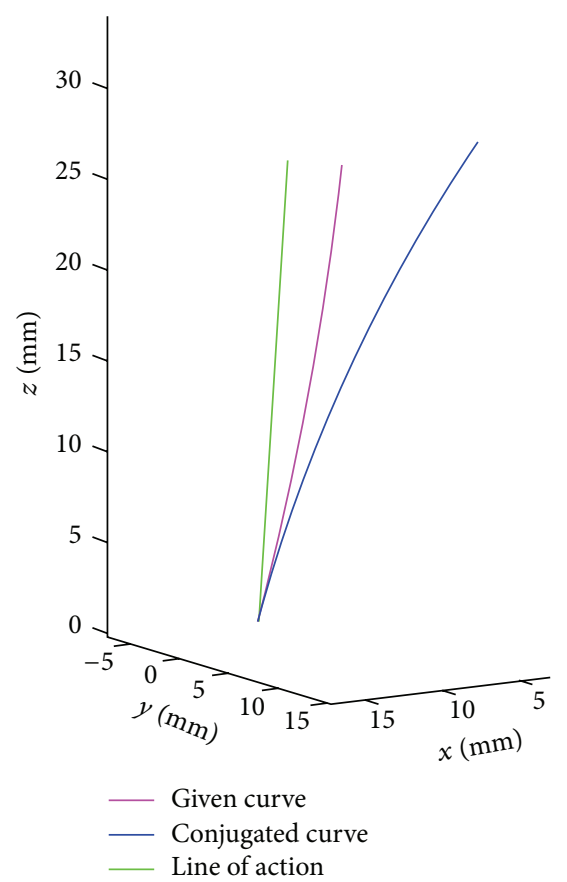

(b)

FIGURE 12: Conjugate spiral curves for parallel axis gear drive.

TABLE 1: Design parameters for conjugate curves.

\begin{tabular}{lc}
\hline Parameters & Values \\
\hline Radius of pitch circle $R / \mathrm{mm}$ & 24 \\
Tooth number of pinion $1 Z_{1}$ & 6 \\
Tooth number of gear $2 Z_{2}$ & 30 \\
Transmission ratio $i_{21}$ & 5 \\
Module $m_{n} / \mathrm{mm}$ & 4 \\
Helix parameter $p$ & 28.6478 \\
Central distance $a / \mathrm{mm}$ & 144 \\
Tooth width $B / \mathrm{mm}$ & 50 \\
Parameter of spiral curve $\theta / \mathrm{rad}$ & $0 \sim 1.0472$ \\
Parameter $t_{1}$ & -0.5 \\
Parameter $t_{2}$ & -0.866 \\
\hline
\end{tabular}

of designated spiral curve expressed in (30) for general cylindrical gears is carried out and the conjugated curve is derived as (31).

Here

$$
R_{1}=R-a ; \quad \theta_{1}=i_{21} \theta .
$$

Then (31) can be written as

$$
\begin{aligned}
& x_{2}=R_{1} \cos \theta_{1}, \\
& y_{2}=-R_{1} \sin \theta_{1}, \\
& z_{2}=p \theta .
\end{aligned}
$$

It is consistent with general form of cylindrical spiral curve in parallel axis gear drive.
4.2.2. Gear Pair with Intersecting Axis. Assuming that parameters $a=0$ and $\Sigma \neq 0$, the gear pair with intersecting axis can be obtained, as shown in Figure 13. The selected conical spiral curve for bevel gears is expressed in coordinate system $S_{1}$, and its parametric form is represented as

$$
\mathbf{r}_{1}=\left(R_{1 f}-c \theta\right) \cos \theta \mathbf{i}_{1}+\left(R_{1 f}-c \theta\right) \sin \theta \mathbf{j}_{1}+p \theta \mathbf{k}_{1},
$$

where $R_{1 f}$ is the radius of pitch circle and $\theta$ and $p$ are also the spiral curve and helix parameters, respectively. It has the correlation $c=p \tan \delta ; \delta$ is the conical angle.

Through a series of calculation on the basis of principle of conjugate curves, its conjugated curve is expressed as

$$
\begin{aligned}
& x_{2}= x_{1}\left[\cos \phi_{1} \cos \left(i_{21} \phi_{1}\right)-\sin \phi_{1} \sin \left(i_{21} \phi_{1}\right) \cos \Sigma\right] \\
&+y_{1}\left[-\sin \phi_{1} \cos \left(i_{21} \phi_{1}\right)-\cos \phi_{1} \sin \left(i_{21} \phi_{1}\right) \cos \Sigma\right] \\
&-z_{1} \sin \left(i_{21} \phi_{1}\right) \sin \Sigma \\
& y_{2}= x_{1}\left[\cos \phi_{1} \sin \left(i_{21} \phi_{1}\right)+\sin \phi_{1} \cos \left(i_{21} \phi_{1}\right) \cos \Sigma\right] \\
&+y_{1}\left[-\sin \phi_{1} \sin \left(i_{21} \phi_{1}\right)+\cos \phi_{1} \cos \left(i_{21} \phi_{1}\right) \cos \Sigma\right] \\
&+z_{1} \cos \left(i_{21} \phi_{1}\right) \sin \Sigma \\
& z_{2}=-x_{1} \cos \phi_{1} \sin \Sigma-y_{1} \sin \phi_{1} \sin \Sigma+z_{1} \cos \Sigma \\
& U \cos \phi_{1}-V \sin \phi_{1}=W .
\end{aligned}
$$




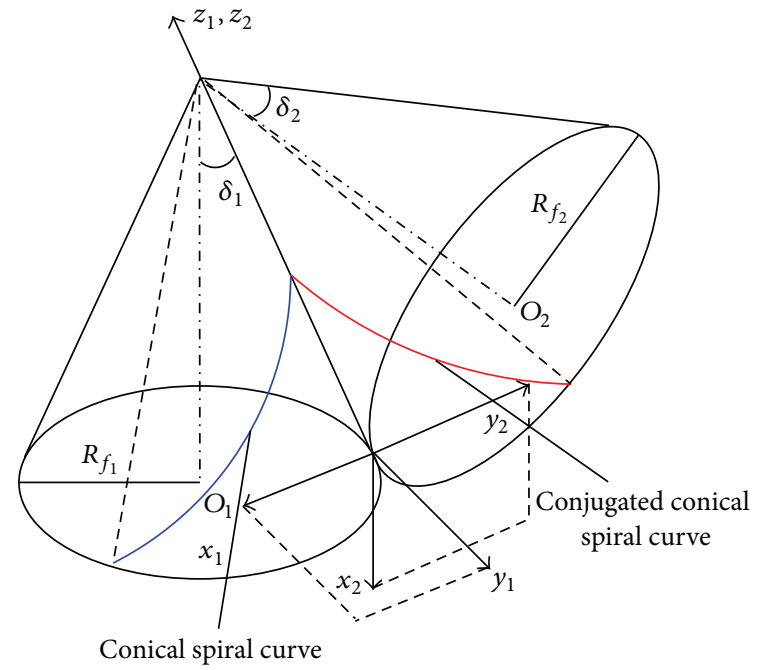

(a)

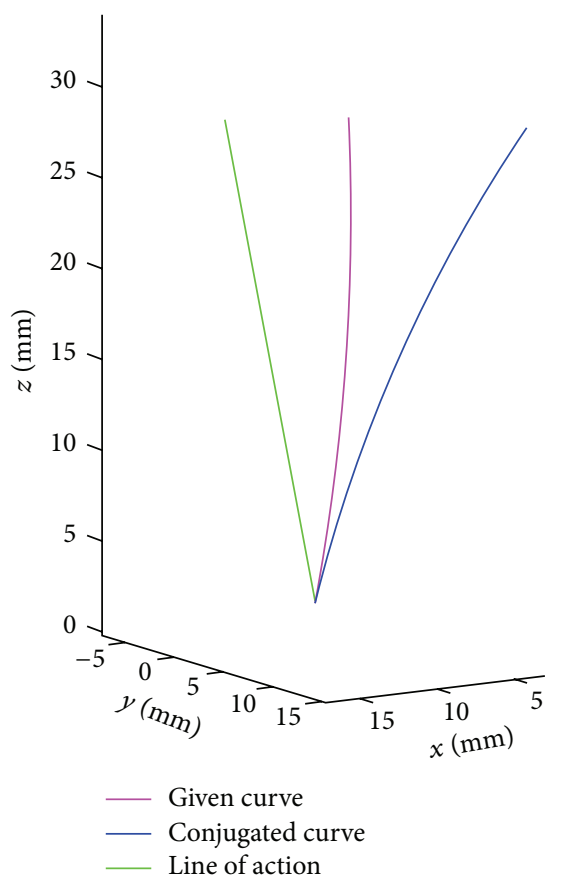

(b)

FIGURE 13: Conjugate spiral curves for intersecting axis gear drive.

Furthermore, substituting (34) into (35), the simplified result with parameter $\theta$ is represented as

$$
\begin{aligned}
& x_{2}=\left[R_{1 f}-(c+p) \theta\right] \cos \left(i_{21} \theta+2 \theta+\Sigma\right), \\
& y_{2}=\left[R_{1 f}-(c+p) \theta\right] \sin \left(i_{21} \theta+2 \theta+\Sigma\right), \\
& z_{2}=(-c \sin \Sigma+p \cos \Sigma) \theta-R_{1 f} \sin \Sigma .
\end{aligned}
$$

Here

$$
\begin{gathered}
c_{1}=c+p ; \quad \theta_{2}=i_{21} \theta+2 \theta+\Sigma \\
p_{1}=-c \sin \Sigma+p \cos \Sigma .
\end{gathered}
$$

Then (36) can be written as

$$
\begin{aligned}
& x_{2}=\left(R_{1 f}-c_{1} \theta\right) \cos \theta_{2}, \\
& y_{2}=\left(R_{1 f}-c_{1} \theta\right) \sin \theta_{2}, \\
& z_{2}=p_{1} \theta-R_{1 f} \sin \Sigma .
\end{aligned}
$$

Conjugated curve attached to gear 2 is got according to above derivation. Obviously, it is also a conical spiral curve in form.

4.2.3. Gear Pair with Crossed Axis. Assuming that parameters $a \neq 0$ and $\Sigma \neq 0$, it gets the gear pair with crossed axis which is depicted in Figure 14. The general spiral curve selected for worm gear drive is given and it has the same expression with (30). Based on the principle of conjugate curves, its conjugated curve is derived as

$$
\begin{aligned}
& x_{2}=\sqrt{p^{2} \sin ^{2} \Sigma+(R-a)^{2}} \cos \left[i_{21} \theta+\arctan \left(\frac{R-a}{p \sin \Sigma}\right)\right], \\
& y_{2}=\sqrt{p^{2} \sin ^{2} \Sigma+(R-a)^{2}} \sin \left[i_{21} \theta+\arctan \left(\frac{R-a}{p \sin \Sigma}\right)\right], \\
& z_{2}=p \theta \cos \Sigma .
\end{aligned}
$$

Here

$$
\begin{gathered}
R_{2}=\sqrt{p^{2} \sin ^{2} \Sigma+(R-a)^{2}} \\
\theta_{3}=i_{21} \theta+\arctan \left(\frac{R-a}{p \sin \Sigma}\right) .
\end{gathered}
$$

Then (39) can be written as

$$
\begin{aligned}
& x_{2}=R_{2} \cos \theta_{3}, \\
& y_{2}=R_{2} \sin \theta_{3}, \\
& z_{2}=p \theta \cos \Sigma .
\end{aligned}
$$

It also represents the similar relationship with given original curve in form.

From what are discussed above, we can conclude that the conjugated curves corresponding to the given spiral curves for various gear axes are still the spiral curves. It can be written as the same expression form with the given 


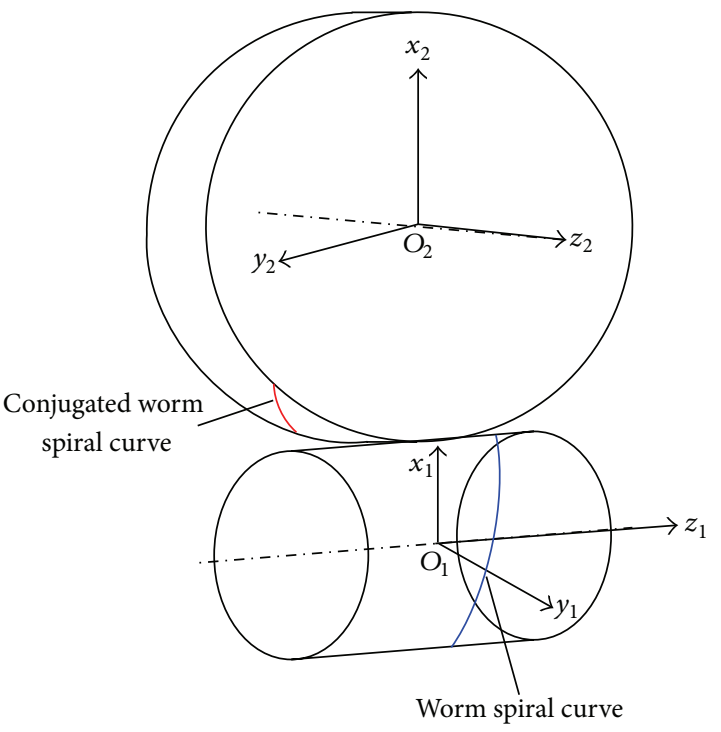

(a)

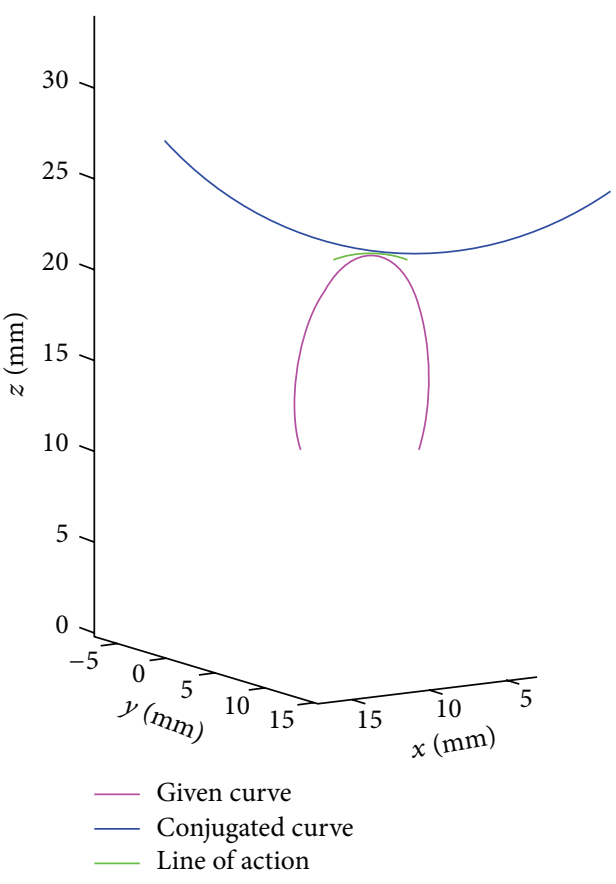

(b)

FIgURE 14: Conjugate spiral curves for crossed axis gear drive.

conditions. It is called meshing commonality of conjugate curves. Moreover, based on the principle of conjugate curves, for any chosen contact curve, the corresponding same type of conjugated curve in the direction of arbitrary contact angle can be identified. Freedom of selecting contact curve suggests the possibility of optimal conjugation design.

According to the aforementioned research, it offers a versatile way for high-performance profile design: (1) few teeth number and large module may be obtained without tooth undercutting. The problems of limited installation space and lightweight design can be solved. (2) Three different contact models of tooth profiles can be established based on the proposed study. Particularly, the special meshing of generated convex and concave tooth profiles makes relative radius of curvature of the contact point longer and increases the contact strength. The load capacity and useful life has been evidently improved. (3) The tooth surfaces mesh in point contact along the conjugate curves and contain the transmission properties of conjugate curves. Due to the selection and generation processes, the approximate pure rolling contact between mating tooth surfaces maybe occurs. The transmission efficiency will be improved.

The further studies on strength property, manufacturing key technology, and performance experiment of conjugatecurve gear drive will be carried out. And the excellent transmission performance of gearing is expected to be obtained on the basis of theoretical and experimental investigations.

\section{Conclusions}

(1) The principle descriptions of conjugate curves for arbitrary axial position are introduced. With the aid of given plane or space curve, generation principle and mathematical model of conjugated curve are developed. It can be applied to parallel axis, intersecting axis, and crossed axis gear drive, respectively.

(2) Based on differential geometry, geometric characteristic analysis of conjugate curves is carried out. The tangent and normal to conjugate curves in arbitrary contact direction are discussed and general calculation methods are provided. The enveloping condition of family of conjugate curves with single parameter is analyzed and characteristic point is determined. Curvature and torsion relationships of conjugate curves are also derived.

(3) Meshing properties of conjugate curves are further revealed. According to a given plane or spatial curve, the variation law of meshing function is analyzed. The uniqueness of conjugated curves is discussed. The conjugated curves are always the same and unique curves for any given curve under different contact angles, while the difference is that there exists the rotational angle relationship with contact point $M$ among generated curves.

(4) Meshing commonality of conjugate curves is demonstrated in terms of a class of spiral curves contacting in the given direction for various gear axes. For any chosen contact curve under various gear axes, the conjugated curve has the same type and it can be written as the same expression form.

(5) The proposed theory lays the foundation for design of new types of gear drive. The further study on conjugate curves and applications in gear transmission will 
be carried out. Excellent transmission performance of gearing is expected to be obtained.

\section{Conflict of Interests}

The authors declare that there is no conflict of interests regarding the publication of this paper.

\section{Acknowledgments}

This project is supported by National Key Technology R\&D Program of the Twelfth Five-year Plan of China (Grant no. 2013BAF01B04), National Natural Science Foundation of China (Grant no. 51205425), and Science and Technology Project of Chongqing City Board of Education (Grant no. KJ122202). The authors also sincerely appreciate the comments and modification suggestions made by the editors and anonymous referees.

\section{References}

[1] S. S. Chern, W. H. Chen, and K. S. Lam, Lectures on Differential Geometry, World Scientific, Singapore, 2006.

[2] P. G. Ciarlet and T.-T. Li, Differential Geometry: Theory and Applications, World Scientific, Singapore, 2007.

[3] F. L. Litvin and A. Fuentes, Gear Geometry and Applied Theory, Cambridge University Press, New York, NY, USA, 2nd edition, 2004.

[4] S. P. Radzevich, Theory of Gearing: Kinetics, Geometry, and Synthesis, CRC Press, Boca Raton, Fla, USA, 2012.

[5] D. B. Dooner, Kinematic Geometry of Gearing, John Wiley \& Sons, 2nd edition, 2012.

[6] F. L. Litvin, A. Fuentes, A. Demenego, D. Vecchiato, and Q. Fan, "New developments in the design and generation of gear drives," Proceedings of the Institution of Mechanical Engineers, Part C: Journal of Mechanical Engineering Science, vol. 215, no. 7, pp. 747-757, 2001.

[7] C. H. Chen, Theory of Conjugate Surfaces and Its Applications, Science and Technology Press, Beijing, China, 2008.

[8] G. X. Li, Spatial Geometry Modeling and Its Application in Engineering, Higher Education Press, Beijing, China, 2007.

[9] F. Di Puccio, M. Gabiccini, and M. Guiggiani, "Generation and curvature analysis of conjugate surfaces via a new approach," Mechanism and Machine Theory, vol. 41, no. 4, pp. 382-404, 2006.

[10] D. R. Wu and J. S. Luo, A Geometric Theory of Conjugate Tooth Surface, World Scientific Publishing, Singapore, 1992.

[11] N. Ito and K. Takahashi, "Differential geometrical conditions of hypoid gears with conjugate tooth surfaces," ASME Journal of Mechanical Design, vol. 122, no. 3, pp. 323-330, 2000.

[12] D. B. Dooner, "On the three laws of gearing," Journal of Mechanical Design, Transactions of the ASME, vol. 124, no. 4, pp. 733-744, 2002.

[13] Z. Duan, H. Chen, and J. Liu, "Principle and transmission technology of Bertrand conjugate surfaces," Chinese Journal of Mechanical Engineering, vol. 19, no. 4, pp. 600-604, 2006.

[14] H. Chen, Z. Duan, J. Liu, and H. Wu, "Research on basic principle of moulding-surface conjugation," Mechanism and Machine Theory, vol. 43, no. 7, pp. 791-811, 2008.
[15] H. Zhang, L. Hua, and X. H. Han, "Computerized design and simulation of meshing of modified double circular-arc helical gears by tooth end relief with helix," Mechanism and Machine Theory, vol. 45, no. 1, pp. 46-64, 2010.

[16] C. F. Chen and C. B. Tsay, "Tooth profile design for the manufacture of helical gear sets with small numbers of teeth," International Journal of Machine Tools and Manufacture, vol. 45, no. 12-13, pp. 1531-1541, 2005.

[17] R. Imin and M. Geni, "Stress analysis of gear meshing impact based on SPH method," Mathematical Problems in Engineering, vol. 2014, Article ID 328216, 7 pages, 2014.

[18] D. Park and A. Kahraman, "A surface wear model for hypoid gear pairs," Wear, vol. 267, no. 9-10, pp. 1595-1604, 2009.

[19] J. Wang, L. Hou, S. M. Luo, and R. Y. Wu, "Active design of tooth profiles using parabolic curve as the line of action," Mechanism and Machine Theory, vol. 67, no. 9, pp. 47-63, 2013.

[20] Q. B. Wang, P. Hu, Y. M. Zhang et al., "A model to determine mesh characteristics in a gear pair with tooth profile error," Advances in Mechanical Engineering, vol. 2014, Article ID 751476, 10 pages, 2014.

[21] C. F. Chen and C. B. Tsay, "Computerized tooth profile generation and analysis of characteristics of elliptical gears with circular arc teeth," Journal of Materials Processing Technology, vol. 148, no. 2, pp. 226-234, 2004.

[22] B. Chen, D. Liang, and Y. E. Gao, "The principle of conjugate curves for gear transmission,” Journal of Mechanical Engineering, vol. 50, no. 1, pp. 130-136, 2014.

[23] B. K. Chen, Y. E. Gao, and D. Liang, "The generation principle of tooth surfaces of conjugate-curve gear transmission," Journal of Mechanical Engineering, vol. 50, no. 3, pp. 18-24, 2014.

[24] D. Liang, B. K. Chen, and Y. E. Gao, "The generation principle and mathematical model of a new involute-helix gear drive," Proceedings of the Institution of Mechanical Engineers, Part C: Journal of Mechanical Engineering Science, vol. 227, no. 12, pp. 2834-2843, 2013.

[25] B. Chen, D. Liang, and Z. Li, "A study on geometry design of spiral bevel gears based on conjugate curves," International Journal of Precision Engineering and Manufacturing, vol. 15, no. 3, pp. 477-482, 2014. 


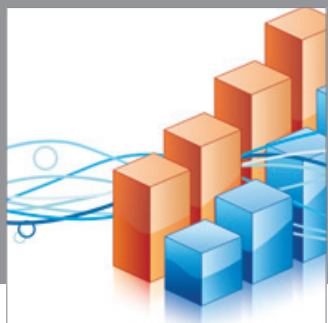

Advances in

Operations Research

mansans

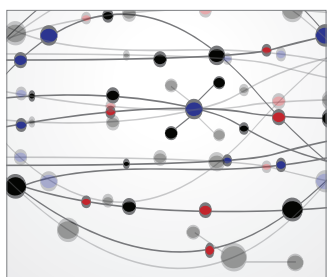

The Scientific World Journal
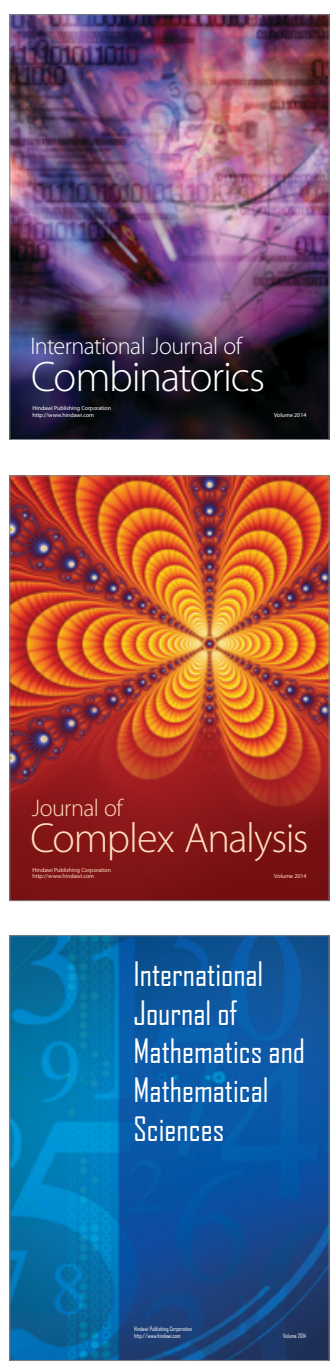
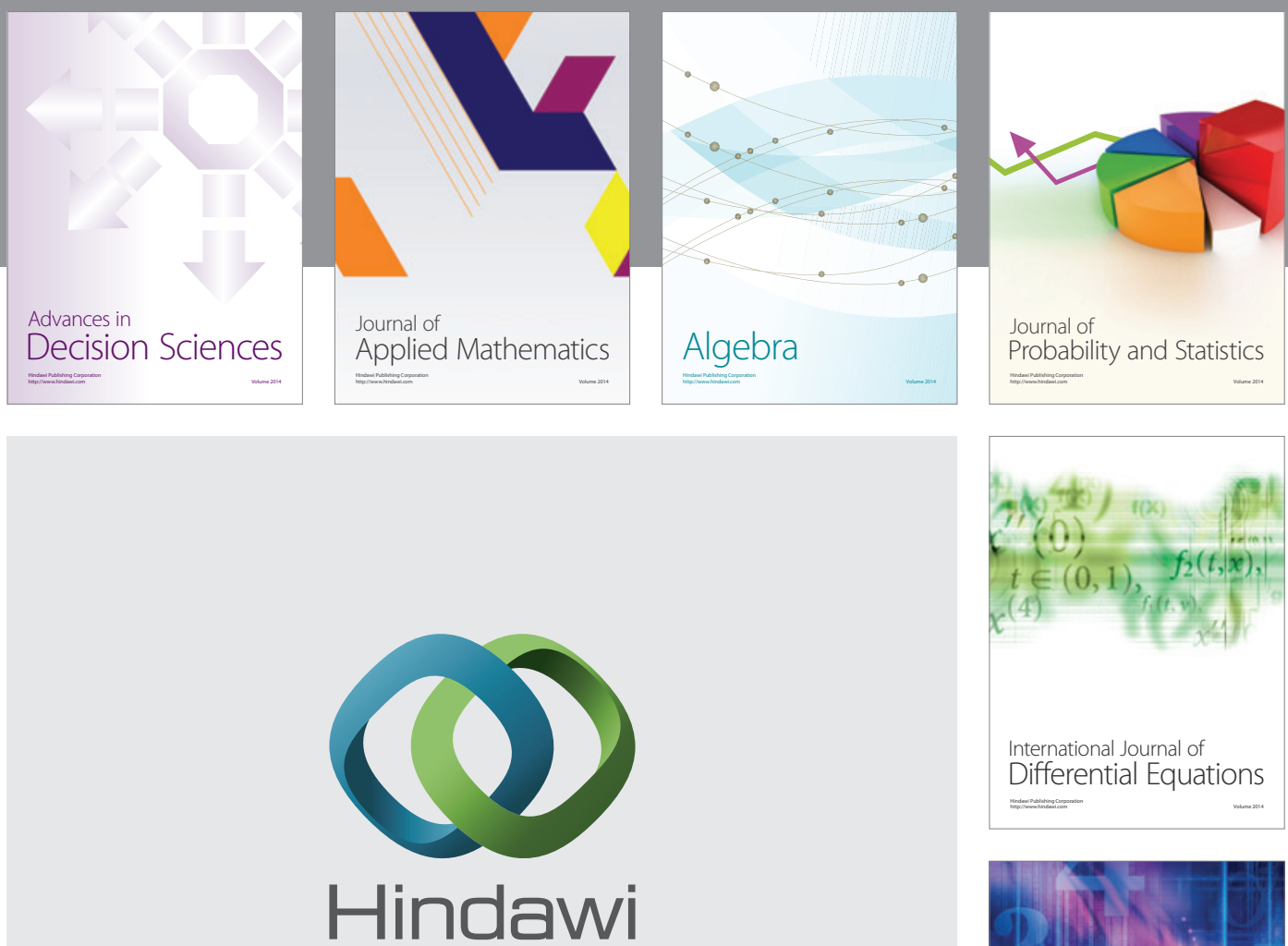

Submit your manuscripts at http://www.hindawi.com
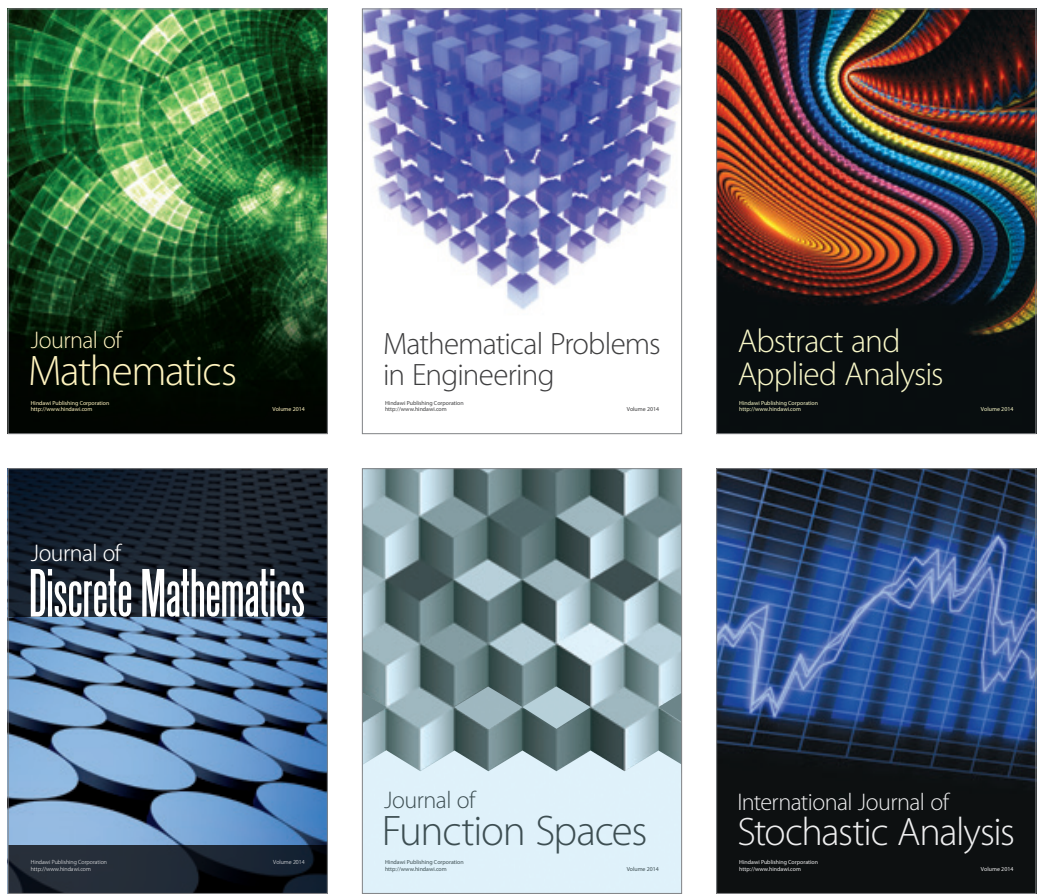

Journal of

Function Spaces

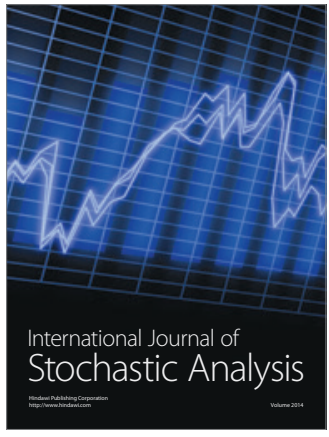

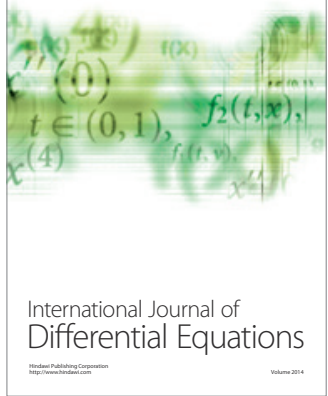
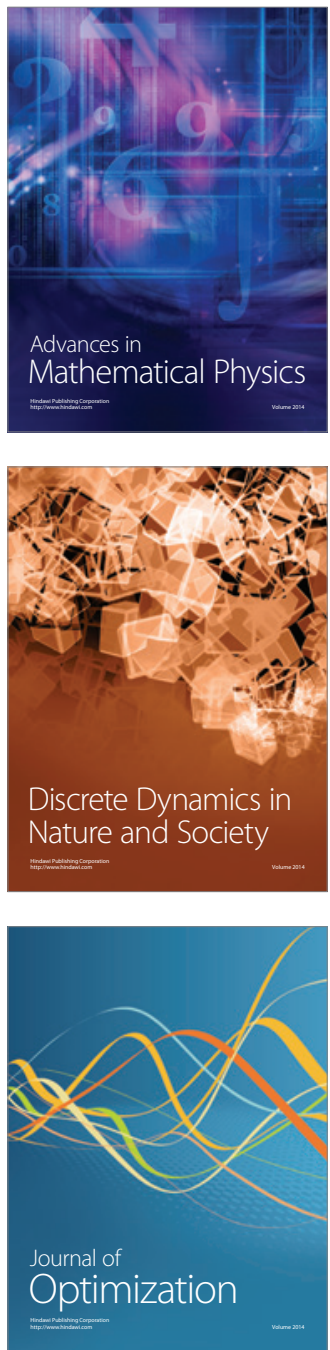